\title{
Adubação foliar nitrogenada e boratada na qualidade da fibra do algodão colorido (Gossypium hirsutum L.)
}

\author{
Effects of nitrogen and boron foliar fertilization on the quality \\ of colored cotton (Gossypium hirsutum L.) fibers
}

'Faculdade Roraimense de Ensino Superior (FARES), Boa Vista, RR, Brasil ${ }^{2}$ Centro de Ciências Agrárias, Universidade Federal da Paraíba (UFPB), Areia, PB, Brasil

*autor correspondente 凹 edgley_agro2008@hotmail.com

Edgley Soares Silva1 *, Diego Almeida Medeiros², Jefferson Alves Dias², Altamiro Oliveira Malta², Samuel Inocêncio Alves da Silva²

RESUMO: O setor têxtil é um dos mais importantes da economia brasileira, congrega mais de 30 mil empresas e emprega aproximadamente 1 milhão de trabalhadores. Objetivou-se, com este trabalho, avaliar o efeito de aplicações foliares de nitrogênio e boro a partir do florescimento sobre a qualidade da fibra do algodão colorido. O experimento foi instalado em ambiente protegido localizado no Departamento de Solos e Engenharia Rural, no município de Areia-PB. O delineamento experimental utilizado foi o inteiramente casualizado. Os fatores avaliados foram quatro épocas de aplicação de adubo foliar, a partir do florescimento (início do florescimento [antese], 3, 5, e 7 semanas após o florescimento) e três tipos de adubação foliar (nitrogenada, boratada e nitrogenada-boratada), com três repetições, distribuídos em esquema fatorial $4 \times 3+1$, totalizando 13 tratamentos. O cultivar utilizado foi o BRS rubi. As aplicações foliares foram realizadas com atomizadores individualizados por tratamento, tendo como fonte o nitrato de amônio e o ácido bórico. As variáveis analisadas foram comprimento de fibras, uniformidade de fibras, índice de fibras curtas, resistência, alongamento à ruptura, índice micronaire, maturidade, reflectância, grau de amarelo e índice de fiabilidade. A adubação foliar nitrogenada, boratada e a frequência de aplicação e suas interações interferem na qualidade da fibra de Gossypium hirsutum L.; Os melhores valores de comprimento, resistência, grau de amarelo de fibras, foram obtidos com um quantitativo de $1,25 \mathrm{~kg} \mathrm{ha}^{-1}$ de boro.

PALAVRAS-CHAVE: BRS rubi, frequência de aplicação, têxtil.
ABSTRACT: The textile industry is a important branch of the Brazilian economy, bringing together more than 30.000 companies and employing approximately 1 million workers. The study aimed to evaluate the effect of foliar applications of nitrogen and boron from flourishing on the quality colored cotton fibers. The experiment was conducted in a greenhouse located in the Department of Soil Science and Agricultural Engineering in Areia-PB. The experimental design was completely randomized. We evaluated four seasons of foliar fertilizer application, from flowering (start of flowering (anthesis) to 3, 5, and 7 weeks after flowering, and three kinds of foliar fertilizer (nitrogen, boron, nitrogen and boron), with three replications, arranged in a $4 \times 3+1$ factorial, totaling 13 treatments. We used BRS rubi cultivar. Foliar applications were made with atomizers for individualized treatment, having as source ammonium nitrate and boric acid. The variables were: fiber length, fiber uniformity, short fiber content, strength, elongation at break, micronaire, maturity, reflectance, color grade and reliability index. Boron nitrogen foliar fertilizationthe frequency of application and their interactions affect the quality of Gossypium hirsutum L. fiber; The best values length, strength, degree of yellow fibers were obtained in a quantitative of $1.25 \mathrm{~kg} \mathrm{ha}^{-1}$ of boron.

KEYWORDS: BRS rubi, frequency of application, textile.

\section{Introdução}

O cultivo e uso da fibra de algodão pelo homem remonta há séculos, com os primeiros fragmentos de tecido datando mais de sete mil anos. Há sólidos indícios de que populações 
ancestrais do Vale do Nilo, no Egito, e do Peru eram bastante familiarizadas com essa cultura. Atualmente, tem crescido o interesse no cultivo do algodão de fibra colorida no mundo e também no Brasil, especialmente pela agricultura familiar, tanto em manejo convencional quanto orgânico (MELCHIORI et al., 2013).

O setor têxtil é um dos mais relevantes da economia brasileira, e em 2012 o valor das vendas industriais dos segmentos e de confecções foi de R \$ 46,5 bilhões. Esses dois elos da cadeia empregaram pouco mais de 1 milhão pessoas. No varejo, o nível de emprego em 2012 foi de 670 mil pessoas. Ressalta-se também que o gasto estimado anual das famílias brasileiras com esses produtos em 2012 foi da ordem de R\$ 102 bilhões (ASSOCIAÇÃO..., 2015).

$\mathrm{O}$ cultivo do algodão colorido na metade do século $\mathrm{XX}$ estava em declínio por causa da baixa produtividade, falta de uniformidade de cores e pobres características, como fibras de menor comprimento, menos fortes e mais baixos índices de finura (SOUSA, 2013).

De acordo com Villari (2010), dentre as diversas características tecnológicas da fibra de interesse da indústria têxtil, tanto para o rendimento industrial quanto para a qualidade do produto final, as mais importantes são: comprimento, uniformidade de comprimento, maturidade, espessura e resistência.

A produtividade média do algodão brasileiro tem crescido em função da utilização de cultivares mais produtivos, maior rendimento de beneficiamento e programas de adubação mais eficientes (SOUZA et al., 2010). Segundo Rosolem, Guaggio e Silva (2001), a adubação adequada regulariza o ciclo e o tamanho das plantas, aumenta o peso médio dos capulhos e das sementes e melhora certas qualidades da fibra, como o comprimento e a maturidade.

Os efeitos da adubação nitrogenada sobre as variáveis de crescimento do algodoeiro destacam-se pela influência sobre o desenvolvimento, precocidade e produtividade da cultura. Além disso, contribui para melhorar os aspectos de qualidade da fibra, sendo, portanto, alguns dos motivos que levam à investigação com esse macronutriente (FERRARI et al., 2012).

Dentre os micronutrientes exigidos pelo algodoeiro, o boro é o mais importante, em virtude de os solos cultivados serem, em sua maioria, pobres em matéria orgânica, principal fonte de boro no solo, e, também, pelo fato de sua disponibilidade ser reduzida com a elevação de $\mathrm{pH}$ após a calagem. Por isso, a manutenção da matéria orgânica do solo, uma calagem adequada e a utilização de adubos contendo boro tornam-se essenciais para o cultivo do algodão no Brasil (MEDEIROS et al., 2003).

No semiárido nordestino, a cultura do algodão herbáceo foi e continua sendo uma das principais atividades do meio rural, em especial dos pequenos e médios produtores. Os maiores problemas enfrentados pelos agricultores é a escassez de água e a falta de recursos para a compra de fertilizantes (EMPRESA..., 2004). Um programa eficiente de adubação voltado para essa cultura seria uma ferramenta fundamental a ser utilizada pelo produtor.

Diante do exposto, o objetivo deste estudo foi avaliar o efeito de aplicações foliares de nitrogênio e boro a partir do florescimento sobre a qualidade da fibra do algodão colorido.

\section{Material e Métodos}

$\mathrm{O}$ experimento foi instalado em ambiente protegido nas dependências do Departamento de Solos e Engenharia Rural (DSER), do Centro de Ciências Agrárias (CCA), da Universidade Federal da Paraíba (UFPB), no município de Areia-PB.

O solo utilizado foi classificado um Latossolo Amarelo de textura argilo-arenosa, coletado na camada de 0 a $20 \mathrm{~cm}$ de profundidade, proveniente do horizonte A (EMPRESA..., 1999). Após a coleta, o solo foi conduzido ao setor de análises pertencente ao Departamento de Solos e Engenharia Rural (DSER/CCA), cujos resultados estão dispostos nas Tabelas 1 e 2.

O delineamento experimental utilizado foi o inteiramente casualizado (DIC). Os fatores avaliados foram quatro épocas de aplicação de adubo foliar, a partir do florescimento (início do florescimento [antese], 3, 5, e 7 semanas após o florescimento) e três tipos de adubação foliar (nitrogenada, boratada e nitrogenada-boratada), com três repetições e uma planta por unidade experimental (composta por vasos plásticos de $8,0 \mathrm{dm}^{-3}$ de capacidade), distribuídos em esquema fatorial $4 \times 3+1$, mais um adicional que foi a testemunha, totalizando 13 tratamentos

Tabela 1. Resultado da análise química do solo, coletado na profundidade de 0 a $20 \mathrm{~cm}$.

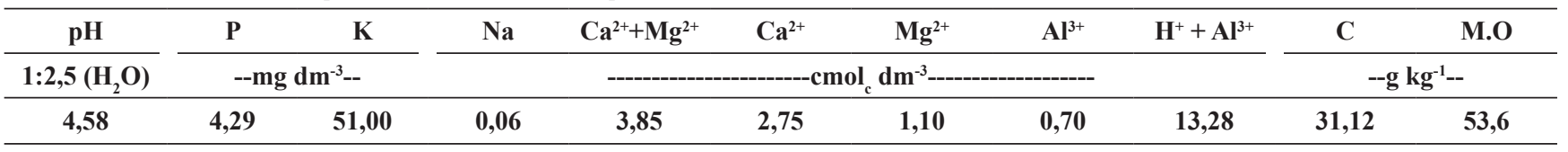

Tabela 2. Resultado da análise física do solo, coletado na profundidade de 0 a $20 \mathrm{~cm}$

\begin{tabular}{|c|c|c|c|c|c|c|c|c|}
\hline \multicolumn{2}{|c|}{ Areia } & \multirow{2}{*}{ Silte } & \multirow{2}{*}{ Argila } & \multirow{2}{*}{ Ad } & \multirow{2}{*}{ Gf } & \multirow{2}{*}{ Ds } & \multirow{2}{*}{ Dp } & \multirow{2}{*}{ Po } \\
\hline Grossa & Fina & & & & & & & \\
\hline \multicolumn{4}{|c|}{ 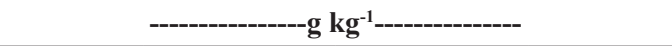 } & \multicolumn{2}{|c|}{----- \%----- } & \multicolumn{2}{|c|}{-----g cm-3 $\mathrm{cm}^{-3----}$} & $--m^{3} m^{-3}--$ \\
\hline 144 & 481 & 43 & 332 & 28 & 91,6 & 1,03 & 2,60 & 0,60 \\
\hline
\end{tabular}

$\mathrm{Ad}=$ argila dispersa $; \mathrm{Gf}=$ grau de floculação; $\mathrm{Ds}=$ densidade do solo; $\mathrm{Dp}=$ densidade de partículas; Po = porosidade . 
Foram semeadas quatro sementes de algodão do cultivar BRS Rubi em vasos plásticos com $6,0 \mathrm{dm}^{-3}$ de solo. Cinco dias após a emergência foi realizado o desbaste, sendo cultivada apenas uma planta por vaso. O solo foi mantido a $80 \%$ da capacidade de retenção de água, através de pesagem e reposição da água perdida.

Os tratamentos foram aplicados no início do florescimento (foi considerado quando $50 \%$ das plantas apresentassem uma flor aberta) da cultura, onde foram realizadas as aplicações dos tratamentos dispostos na Tabela 3.

As aplicações foliares foram realizadas com atomizadores, individualizados por tratamento, tendo como fonte o nitrato de amônio - 35\% de N (solução a 10\%) e o ácido bórico - 17\% de B (solução a 0,5\%), aplicando-se um volume de calda equivalente a $250 \mathrm{~L} \mathrm{ha}^{-1}$ no início do dia.

A fibra foi coletada ao final da sétima semana após o florescimento. As características intrínsecas e extrínsecas das fibras foram determinadas pelo sistema HVI (High Volume Instruments), no qual se medem as principais características físicas definidas pelo USDA (Departamento de Agricultura dos Estados Unidos da América), tanto para o mercado de algodão quanto para o melhoramento genético.

As variáveis estudadas foram: comprimento de fibras, uniformidade de fibras, índice de fibras curtas, resistência, alongamento à ruptura, índice micronaire, maturidade, reflectância, grau de amarelo e índice de fiabilidade.

Os valores obtidos foram submetidos à análise de variância, sendo o nível de significância determinado pelo teste F. Os dados foram analisados pelo sistema para Análises Estatísticas da Universidade Federal de Viçosa (SAEG).

\section{Resultados e Discussão}

É possível observar na Tabela 4 que houve efeito significativo dos tratamentos para todas as variáveis estudadas a $1 \%$ de probabilidade pelo teste F. No entanto, os adubos testados, a frequência de aplicação, as interações adubo x aplicação e o teste $\mathrm{x}$ fatorial não tiveram efeito significativo sobre $\mathrm{o}$ comprimento de fibras. A interação teste $\mathrm{x}$ fatorial não influenciou significativamente sobre a uniformidade de fibras e o índice de fibras curtas pelo teste $\mathrm{F}$.

Para a variável de comprimento de fibra, foi registrada uma média de 26,5 mm com dosagem de $1,25 \mathrm{~kg} \mathrm{ha}^{-1}$ de boro. Lima et al. (2006), testando adubação nitrogenada e bioestimulante aplicado nas folhas do algodoeiro colorido, obtiveram valores um pouco acima (26,4 a $27,5 \mathrm{~mm}$ ) dos obtidos neste estudo. O comprimento da fibra é influenciado principalmente pela variedade, exposição da planta a temperaturas elevadas, estresse hídrico e deficiência nutricional. Resultados semelhantes aos encontrados neste estudo foram observados por Staut e Kurihara (2001), que ao estudarem diversos cultivares de algodoeiro herbáceo, afirmaram que doses adequadas de nitrogênio contribui para maior comprimento das fibras.

Com relação à uniformidade de fibras, os valores obtidos foram de 82,5\% nas interações com N-B $\left(25-1,25 \mathrm{~kg} \mathrm{ha}^{-1}\right)$. Araújo et al. (2013), com objetivo de avaliar o efeito de lâminas de água e de cultivares sobre os componentes da fibra do algodoeiro herbáceo, registraram valores semelhantes $(86,1 \%)$ aos observados no presente estudo. A uniformidade do comprimento afeta a uniformidade do fio e da resistência e a eficiência do processo de fiação que está relacionado com o conteúdo de fibra curta. $\mathrm{O}$ baixo índice de uniformidade pode ter uma elevada percentagem de fibras curtas, dificultando o processamento e produzindo fios de baixa qualidade.

O índice de fibras curtas, no entanto, obteve os maiores valores para o tratamento quando se aplicou a adubação nitrogenada na quantidade de $25 \mathrm{~kg} \mathrm{ha}^{-1}$ (34,3\%). Esses valores estão bem acima dos verificados $(2,69 \%)$ por Azevedo et al. (2005), onde na ocasião testaram o efeito de adubação nitrogenada e irrigação com água residuária tratada.

A resistência da fibra obteve maior média quando se aplicou adubação boratada, fornecendo um quantitativo

Tabela 3. Disposição dos tratamentos aplicados com respectivos adubos e frequências.

\begin{tabular}{|c|c|c|c|c|}
\hline Tratamentos & Adubo & Frequência de aplicação & Adubo aplicado $\left(\mathrm{kg} \mathrm{ha}^{-1}\right)$ & $\begin{array}{l}\text { Número de semanas } \\
\text { após o florescimento }\end{array}$ \\
\hline 1 & $\mathbf{N}$ & 1 & 25 & 1 \\
\hline 2 & $\mathbf{N}$ & 2 & 75 & 1,2 e 3 \\
\hline 3 & $\mathbf{N}$ & 3 & 125 & $1,2,3,4$ e 5 \\
\hline 4 & $\mathbf{N}$ & 4 & 175 & $1,2,3,4,5,6$ e 7 \\
\hline 5 & B & 1 & 1,25 & 1 \\
\hline 6 & B & 2 & 3,75 & 1,2 e 3 \\
\hline 7 & B & 3 & 6,25 & $1,2,3,4$ e 5 \\
\hline 8 & B & 4 & 8,75 & $1,2,3,4,5,6$ e 7 \\
\hline 9 & N-B & 1 & $25-1,25$ & 1 \\
\hline 10 & N-B & 2 & $75-3,75$ & 1,2 e 3 \\
\hline 11 & N-B & 3 & $125-6,25$ & $1,2,3,4$ e 5 \\
\hline 12 & N-B & 4 & $175-8,75$ & $1,2,3,4,5,6$ e 7 \\
\hline 13 & Testemunha & & ----------- & ----------- \\
\hline
\end{tabular}


Tabela 4. Médias da análise de variância das variáveis de comprimento de fibras (UHM), uniformidade de fibras (UNF), índice de fibras curtas (SFI), resistência (STR) e alongamento à ruptura (ELG) do algodoeiro (Gossypium hirsutum L.) em função da adubação foliar com N e B.

\begin{tabular}{|c|c|c|c|c|c|c|c|}
\hline Tratamentos & Adubo & $\begin{array}{l}\text { Frequência de } \\
\text { aplicação }\end{array}$ & $\begin{array}{l}\text { UHM } \\
(\mathrm{mm})\end{array}$ & $\begin{array}{l}\text { UNF } \\
(\%)\end{array}$ & $\begin{array}{l}\text { SFI } \\
(\%)\end{array}$ & $\begin{array}{c}\text { STR } \\
\text { (g/tex) }\end{array}$ & $\begin{array}{c}\text { ELG } \\
(\%)\end{array}$ \\
\hline 1 & $\mathbf{N}$ & 1 & 24,1 & 76,9 & 34,3 & 25,9 & 4,4 \\
\hline 2 & $\mathbf{N}$ & 2 & 24,9 & 78,7 & 17,7 & 20,8 & 5,3 \\
\hline 3 & $\mathbf{N}$ & 3 & 24,9 & 77,9 & 28,8 & 26,7 & 3,9 \\
\hline 4 & $\mathbf{N}$ & 4 & 25,7 & 77,8 & 15,3 & 19,9 & 6,6 \\
\hline 5 & B & 1 & 26,5 & 77,5 & 15,3 & 18,5 & 6,3 \\
\hline 6 & B & 2 & 24,7 & 77,7 & 26,9 & 30,9 & 5,7 \\
\hline 7 & B & 3 & 24,9 & 78,4 & 22,6 & 24,7 & 5,3 \\
\hline 8 & B & 4 & 25,0 & 78,7 & 16,5 & 21,9 & 5,7 \\
\hline 9 & N-B & 1 & 25,7 & 82,5 & 6,7 & 27,4 & 5,5 \\
\hline 10 & N-B & 2 & 25,6 & 80,6 & 4,7 & 23,4 & 5,7 \\
\hline 11 & N-B & 3 & 25,7 & 79,5 & 18,9 & 27,7 & 5,9 \\
\hline 12 & N-B & 4 & 23,9 & 75,9 & 30,3 & 18,5 & 6,7 \\
\hline 13 & \multicolumn{2}{|c|}{ Testemunha } & 24,8 & 78,5 & 20,4 & 27,5 & 6,7 \\
\hline \multirow{6}{*}{ Valor de F } & \multicolumn{2}{|c|}{ Tratamento } & $3,2 * *$ & $22,8 * *$ & $780,8 * *$ & $130,7 * *$ & $7,0 * *$ \\
\hline & \multicolumn{2}{|c|}{ Adubo } & $1,1^{\mathrm{NS}}$ & $30,1 * *$ & $770,2 * *$ & $7,4 * *$ & $8,7 * *$ \\
\hline & \multicolumn{2}{|c|}{ Aplicação } & $0,9^{\mathrm{NS}}$ & $12,6 * *$ & $256,9 * *$ & $173,9 * *$ & $8,7 * *$ \\
\hline & \multicolumn{2}{|c|}{ Adubo x Aplicação } & $1,5^{\mathrm{NS}}$ & $27,8 * *$ & $1102,7 * *$ & $144,6 * *$ & $5,1 * *$ \\
\hline & \multicolumn{2}{|c|}{ Teste x Fatorial } & $0,6^{\mathrm{NS}}$ & $0,3^{\mathrm{NS}}$ & $2,8^{\mathrm{NS}}$ & $101,1 * *$ & $12,1 * *$ \\
\hline & \multicolumn{2}{|c|}{ C.V (\%) } & 2,7 & 0,7 & 2,7 & 2,4 & 9,6 \\
\hline
\end{tabular}

Legendas: $\mathrm{NS}=$ não significativo; $* *$, * significativos a 1 e $5 \%$, pelo teste $\mathrm{F}$.

de 1,25 $\mathrm{kg} \mathrm{ha}^{-1}\left(30,9 \mathrm{~g} / \mathrm{tex}^{-1}\right)$. Jerônimo et al. (2014), avaliando a qualidade da semente e fibra de algodão na caracterização do descaroçador, obtiveram valores abaixo dos registrados nesse trabalho $\left(25,22\right.$ a $\left.27,99 \mathrm{~g} / \mathrm{tex}^{-1}\right)$. A resistência da fibra é determinada pela variedade e pode ser afetada por deficiência nutricional, como também das condições meteorológicas.

Para a variável alongamento à ruptura, a maior média observada foi de $6,7 \%$ para as interações N-B, sendo os valores dos fertilizantes de $175-8,75 \mathrm{~kg} \mathrm{ha}^{-1}$. Esse valor pode ser comparado com os verificados por Araújo et al. (2013), onde na ocasião registraram médias de $4,6 \%$, valor esse abaixo dos obtidos neste estudo.

Com relação à Tabela 5 , houve efeito significativo das variáveis independentes: tratamentos, adubos, aplicação e a interação de adubo $\mathrm{x}$ aplicação para as variáveis dependentes: maturidade, refletância e índice de fiabilidade a $1 \%$ de probabilidade pelo teste $\mathrm{F}$. A interação teste $\mathrm{x}$ fatorial influenciou positivamente as variáveis de grau de amarelo (5\%) e o índice de fiabilidade (1\%).

Em valores absolutos, o índice de micronaire obtido foi de 2,9 $\mu \mathrm{g}$ pol- $^{2}$, com as adubações individuais de B e N. Jerônimo et al. (2014), avaliando a qualidade da fibra de algodão, obtiveram valores acima dos registrados neste trabalho $\left(4,07\right.$ a $4,63 \mu \mathrm{g}$ pol- $\left.{ }^{2}\right)$. As medições de micronaire podem ser influenciadas durante o período de crescimento do algodoeiro pelas condições ambientais, deficiência de nutrientes, como também pelo ataque de pragas e doenças.

A maior média de maturidade $(84,7)$ foi obtida quando se adubou com boro $\left(3,75 \mathrm{~kg} \mathrm{ha}^{-1}\right)$ e com a interação N-B (125-6,25 kg ha-1). Azevedo et al. (2005), estudando as características tecnológicas da fibra do algodão herbáceo sob efeito de adubação nitrogenada, registraram valores superiores aos deste trabalho $(89,25)$.

Para a variável reflectância, observam-se valores de 29,4\% em plantas adubadas com boro, com um quantitativo de $6,25 \mathrm{~kg} \mathrm{ha}^{-1}$. Azevedo et al. (2005) obtiveram valores acima dos registrados neste estudo (79,13\%). Essa variável, por sua vez, indica a quantidade de luz refletida em uma amostra.

No tocante ao grau de amarelo, a maior média registrada foi quando se aplicou individualmente adubação nitrogenada de $125 \mathrm{~kg} \mathrm{ha}^{-1} \mathrm{e}$ boro no quantitativo de $1,25 \mathrm{~kg} \mathrm{ha}^{-1}$, para o valor de 25,4 +b-. Em pesquisa desenvolvida por Lima et al. (2006) obtiveram-se valores abaixo dos pesquisados neste trabalho $(14,2+b-)$. A coloração das fibras pode ser afetada por chuvas, geadas, pragas, doenças, contato da pluma com o solo e grande variação de temperatura durante o armazenamento.

A respeito do índice de fiabilidade, a maior média foi de 2615,8, com a interação dos nutrientes N-B nos quantitativos de 25-1,25 $\mathrm{kg} \mathrm{ha}^{-1}$, respectivamente. Jerônimo et al. (2014) obtiveram um variação de 1520,0 a 2459,1 para essa variável. 
Tabela 5. Médias da análise de variância das variáveis de índice micronaire (MIC), maturidade (MAT), reflectância (Rd), grau de amarelo (+b-) e índice de fiabilidade (CSP) do algodoeiro (Gossypium hirsutum L.) em função da adubação foliar com N e B.

\begin{tabular}{|c|c|c|c|c|c|c|c|}
\hline Tratamentos & Adubo & $\begin{array}{l}\text { Frequência de } \\
\text { aplicação }\end{array}$ & $\begin{array}{c}\text { MIC } \\
\left(\mu \mathrm{g} \mathrm{pol}^{-2}\right)\end{array}$ & MAT & $\begin{array}{l}\text { Rd } \\
(\%)\end{array}$ & $+b-$ & CSP \\
\hline 1 & $\mathbf{N}$ & 1 & 2,4 & 82,7 & 27,6 & 24,5 & 1946,9 \\
\hline 2 & $\mathbf{N}$ & 2 & 2,9 & 83,7 & 28,5 & 24,5 & 1704,9 \\
\hline 3 & $\mathbf{N}$ & 3 & 2,5 & 83,7 & 23,5 & 25,4 & 2078,4 \\
\hline 4 & $\mathbf{N}$ & 4 & 2,7 & 81,7 & 23,7 & 24,9 & 1667,1 \\
\hline 5 & B & 1 & 2,6 & 81,7 & 23,5 & 25,4 & 1592,1 \\
\hline 6 & B & 2 & 3,5 & 84,7 & 28,5 & 24,5 & 2057,1 \\
\hline 7 & B & 3 & 2,7 & 83,7 & 29,4 & 24,5 & 1923,9 \\
\hline 8 & B & 4 & 2,9 & 83,7 & 26,7 & 24,9 & 1769,2 \\
\hline 9 & N-B & 1 & 2,5 & 82,7 & 25,8 & 24,7 & 2615,8 \\
\hline 10 & N-B & 2 & 2,5 & 82,7 & 25,7 & 23,9 & 2170,1 \\
\hline 11 & N-B & 3 & 3,5 & 84,7 & 23,9 & 24,8 & 2114,0 \\
\hline 12 & N-B & 4 & 2,3 & 80,7 & 25,3 & 24,7 & 1404,1 \\
\hline 13 & \multicolumn{2}{|c|}{ Testemunha } & 2,8 & 82,7 & 25,9 & 23,9 & 2069,9 \\
\hline & \multicolumn{2}{|c|}{ Tratamentos } & $1,4^{\mathrm{NS}}$ & $12,5^{* *}$ & $40,3 * *$ & $1,7^{\mathrm{NS}}$ & $292408 * *$ \\
\hline & \multicolumn{2}{|c|}{ Adubo } & $1,2^{\mathrm{NS}}$ & $5,3 * *$ & $33,7 * *$ & $1,2^{\mathrm{NS}}$ & $222065 * *$ \\
\hline & \multicolumn{2}{|c|}{ Aplicação } & $1,8^{\mathrm{NS}}$ & $26,0 * *$ & $33,5 * *$ & $2,3^{\mathrm{NS}}$ & $391863 * *$ \\
\hline Valor de F & \multicolumn{2}{|c|}{ Adubo x Aplicação } & $1,7^{\mathrm{NS}}$ & $10,3 * *$ & $57,4 * *$ & $1,3^{\mathrm{NS}}$ & $306370 * *$ \\
\hline & \multicolumn{2}{|c|}{ Teste $x$ Fatorial } & $0,1^{\mathrm{NS}}$ & $0,9^{\mathrm{NS}}$ & $0,2^{\mathrm{NS}}$ & $4,8^{*}$ & $62685 * *$ \\
\hline & \multicolumn{2}{|c|}{ C.V (\%) } & 19,5 & 0,7 & 2,1 & 2,4 & 0,1 \\
\hline
\end{tabular}

Legendas: NS = não significativo; $* *, *=$ significativos a 1 e $5 \%$, respectivamente pelo teste $\mathrm{F}$.

\section{Conclusões}

A adubação foliar nitrogenada, boratada, e a frequência de aplicação e suas interações, interferem na qualidade da fibra de Gossypium hirsutum L.

Os melhores valores de comprimento, resistência, grau de amarelo de fibras, foram obtidos com um quantitativo de $1,25 \mathrm{~kg} \mathrm{ha}^{-1}$ de boro.

$\mathrm{O}$ alongamento à ruptura, a uniformidade de fibras, o índice de fiabilidade e a maturidade sofreram influência das interações N-B.

O quantitativo de $6,25 \mathrm{~kg} \mathrm{ha}^{-1}$ de boro influencia a reflectância de fibra de $G$. hirsutum L.

A adubação nitrogenada de $25,0 \mathrm{~kg} \mathrm{ha}^{-1}$ influencia o índice de fibras curtas de G. hirsutum L.

Não houve influência das adubações sobre o micronaire da fibra de G. hirsutum L.

\section{Referências}

ARAÚJO, W. P. et al. Componentes da fibra de cultivares de algodoeiro herbáceo sob lâminas de água. Revista Educação Agrícola Superior, Brasília, v. 28, n. 1, p. 78-81, 2013.

ASSOCIAÇÃO BRASILEIRA DE VAREJO TÊXTIL. Notícias: desempenho da indústria têxtil e de confecções. ABVTEX, 2015. Disponível em: $<$ http://www.abvtex.org.br/index.php?option=com_co ntent\&view=article\&id=3731\%3Adesepenho-da-industria-textil- edeconfecco es\&cati $\mathrm{d}=32 \% 3$ Aabvt ex\&Itemid=128\&lang $=\mathrm{pt}>$. Acesso em: 25 jun. 2016.

AZEVEDO, M. R. Q. A. et al. Características tecnológicas da fibra do algodão herbáceo sob efeito de adubação nitrogenada e irrigação com água residuária tratada. Revista Brasileira de Engenharia Agrícola e Ambiental, Campina Grande, v. 9, n. 1, p. 202-206, 2005.

Empresa Brasileira de Pesquisa Agropecuária - EMBRAPA. Algodão colorido no Brasil, e em particular no Nordeste e no Estado da Paraíba. Campina Grande: Embrapa Algodão, 2004. 17 p.

Empresa Brasileira de Pesquisa Agropecuária - EMBRAPA. Sistema brasileiro de classificação dos solos. Rio de Janeiro: Embrapa Solos, 1999. $412 \mathrm{p}$

FERRARI, S. et al. Plantas de cobertura e adubação nitrogenada na cultura do algodoeiro. Revista Brasileira Ciências Agrárias, Recife, v. 7, n. 2, p. 226-232, 2012.

JERÔNIMO, J. F. et al. Qualidade da semente e fibra de algodão na caracterização do descaroçador de 25 serras. Revista Brasileira de Engenharia Agrícola e Ambiental, Campina Grande, v. 18, n. 6, p. 664-671, 2014.

LIMA, M. M. et al. Níveis de adubação nitrogenada e bioestimulante na produção e qualidade do algodão BRS verde. Revista Brasileira de Engenharia Agrícola e Ambiental, Campina Grande, v. 10, n. 3, p. 619-623, 2006.

MEdeIROS, J. F.; BELTRÃO, N. E. M.; MEdEIROS, J. C. Boro no metabolismo vegetal e adubação boratada no algodoeiro herbáceo. Revista Brasileira de Fibras, Campina Grande, v.7, n. 2-3, p. 743-752, 2003. 
MELCHIORI, T. et al. Produtividade de cultivares de algodão colorido no sul de Minas Gerais. In: JORNADA CIENTÍFICA E TECNOLÓGICA, 5., SIMPÓSIO DE PÓS-GRADUAÇÃO DO IFSULDEMINAS, 2., 2013, Inconfidentes. Anais... Inconfidentes: IFSULDEMINAS, 2013. p. 1-5.

ROSOlEM, C. A.; GUAGGIO, J. A.; SILVA, N. M. Algodão, amendoim e soja. In: Ferreira, M. E.; Cruz, M. C. P.; Raij, B. V. \& Abreu, C. A. (Eds.). Micronutrientes e elementos tóxicos na agricultura. Jaboticabal: CNPq, FAPESP, POTAFOS, 2001. p. 319-354.

SOUSA, G. R. M. Aplicação da espectroscopia NIR para análise exploratória de plumas e tecidos de algodão colorido. 2013. 55 f. Dissertação (Mestrado em Ciências Agrárias) - Centro de Ciências Humanas e Agrárias, Universidade Estadual da Paraíba, Paraíba, 2013.

SOUZA, T. A. F. et al. Crescimento do algodão colorido em função da aplicação foliar de N e B. In: CONGRESSO BRASILEIRO DE
MAMONA4., SIMPÓSIO INTERNACIONAL DE OLEAGINOSAS ENERGÉTICAS, 1., 2010, João Pessoa. Anais... Campina Grande: EMBRAPA ALGODÃO, 2010. p. 535-539.

STAUT, L. A.; KURIHARA, C. H. Calagem e adubação. In: EMBRAPA AGROPECUÁRIA. (Eds.). Algodão: tecnologia de produção. Dourados: Embrapa Agropecuária Oeste/Embrapa Algodão, 2001. p. 103-123.

VILLARI, A. C. Guia do algodão: tecnologia no campo para uma indústria de qualidade. Conselho de Informações sobre Biotecnologia, 2010. Disponível em: <http://cib.org.br/wp-content/uploads/2011/10/ GuiaAlgodaoSet2010.pdf>. Acesso em: 14 jun. 2016.

Recebido: 19 jul. 2016 Aprovado: 05 mar. 2018 